\title{
Duct Adenoma of the Breast as Background Process for Cholesterol Granuloma: Case Report and Literature Review
}

\author{
Nina A. Oleynikova, $\mathrm{PhD}^{1^{*}}$; Olga A. Kharlova ${ }^{1}$; Pavel G. Malkov, $\mathrm{PhD}, \mathrm{ScD}^{1}$; \\ Natalia V. Danilova, $\mathrm{PhD}^{1}$; Nikita S. Kharlov²; Sara Y. Davydova ${ }^{1,3}$ \\ ${ }^{1}$ Lomonosov Moscow State University, Moscow, Russia \\ ${ }^{2}$ First Moscow State Medical University, Moscow, Russia \\ ${ }^{3}$ Tel-Aviv Souransky Medical Center, Tel-Aviv, Israel
}

\begin{abstract}
Cholesterol granuloma is an extremely rare benign condition that should be considered in the differential diagnosis of breast lesions suspected of being malignant. We present a case of a 55-year-old woman who presented with a nodule mass in the upper external quadrant of the right breast on a routine mammography examination. The case presented here is the first one where the mammary duct adenoma represents a background process for cholesterol granuloma. The described case is noticeable by the combination of multiple cholesterol granulomas in the lungs with breast presentation, which was associated ductal adenoma. (International Journal of Biomedicine. 2018;8(1):69-74.)
\end{abstract}

Key Words: cholesterol granuloma $\bullet$ breast $\bullet$ lung $\bullet$ duct adenoma of the breast

\section{Introduction}

Cholesterol granuloma is an extremely rare benign condition that should be considered in the differential diagnosis of breast lesions suspected of being malignant. There is no difficulty in distinguishing cholesterol granuloma from a breast carcinoma on histological examination; however, the clinical findings and radiographic images of cholesterol granuloma are similar to those of cancer, leading to possible confusion between these two diseases. ${ }^{(1)}$ The pathogenesis of breast cholesterol granuloma is still poorly understood and remains controversial. Since 1974 only 20 cases of cholesterol granulomahave been described as a background process for mammary duct ectasia. ${ }^{(2-6)}$ However, in 2004, C. Furuhira et colleagues reported a case of breast cholesterol granuloma accompanied by cancer. ${ }^{(7)}$

\section{Case Presentation}

We present a case of a 55 -year-old woman who presented with a nodule mass in the upper external quadrant of the right

*Corresponding author: Nina A. Oleynikova, PhD. Staff Scientist, Department of Pathology, Lomonosov Moscow State University, the Russian Federation.E-mail: ale x_05@mail.ru breast on a routine mammography examination. Physical examination revealed a palpable nodule $(\mathrm{d}=2 \mathrm{~cm})$ in the upper external quadrant of the right breast that was elastic, firm, and mobile, with ill-defined margins and without associated skin symptoms or regional lymph node involvement. Contralateral breast examination has not revealed any pathological findings.

Blood test: $\mathrm{Hb}-143 \mathrm{~g} / \mathrm{L}, \mathrm{RBC}-4.87 \times 10^{12} / \mathrm{L}, \mathrm{WBC}$ - $6.6 \times 10^{9} / \mathrm{L}$, lymphocytes $-41.2 \%$, monocytes $-4.8 \%$, eosinophils $-2.0 \%$, basophils $-0.5 \%$, platelets $-179 \times 10^{9} / \mathrm{L}$, $\mathrm{ESR}-5 \mathrm{~mm} / \mathrm{h}$.

Blood chemistry: total cholesterol $-6.58 \mathrm{mmol} / \mathrm{L}$; triglycerides $-3.36 \mathrm{mmol} / \mathrm{L}$; urea $-463 \mu \mathrm{mol} / \mathrm{L}$; total bilirubin $-21.2 \mu \mathrm{mol} / \mathrm{L}$.

Chest CT: In S3 of the right lung and in the $\mathrm{C} 4$ of the left lung, the dense foci with clear contours are visualized.

An ultrasonogram of the right breast disclosed an irregularly shaped mass $35 \times 18 \mathrm{~mm}$ with multiple, floating, hyperechoic specks within the cyst and ill-defined margins (Fig. 1e-f).

An MSCT of the chest showed a solid nodule with indistinct tuberous margins $23 \times 15 \times 13 \mathrm{~mm}$ without enlargement of the axillar nodules or bone distraction (Fig. 1a-d).

Cytological examination of a specimen obtained by fine needle aspiration biopsy presented as a small piece of breast tissue with solitary ducts and lobules. More detailed 
examination of lobules revealed epithelial proliferation and nuclei enlargement, which was considered as a carcinoma in situ with tentative diagnosis of ductal carcinoma (Fig. 2e-f). Based on that, a lumpectomy of the right breast was performed.

Gross examination of a surgical specimen measuring $10.5 \times 7 \times 2.5 \mathrm{~cm}$ showed an irregular shaped mass with illdefined margins $1.8 \times 1 \times 2.3 \mathrm{~cm}$ at $0.8 \mathrm{~cm}$ from the fascial margin of resection. An oyster white mass with brown patterns was revealed on section. Microscopic examination showed ducts and lobules with epithelial hyperplasia and apocrine metaplasia, which had formed intraductal papillary structures (Fig. 2a-d). A great mass of fibroadipose tissue was found in breast stroma containing cholesterol granuloma composed predominantly of cholesterol clefts, some of which were surrounded by multinucleated giant cells.

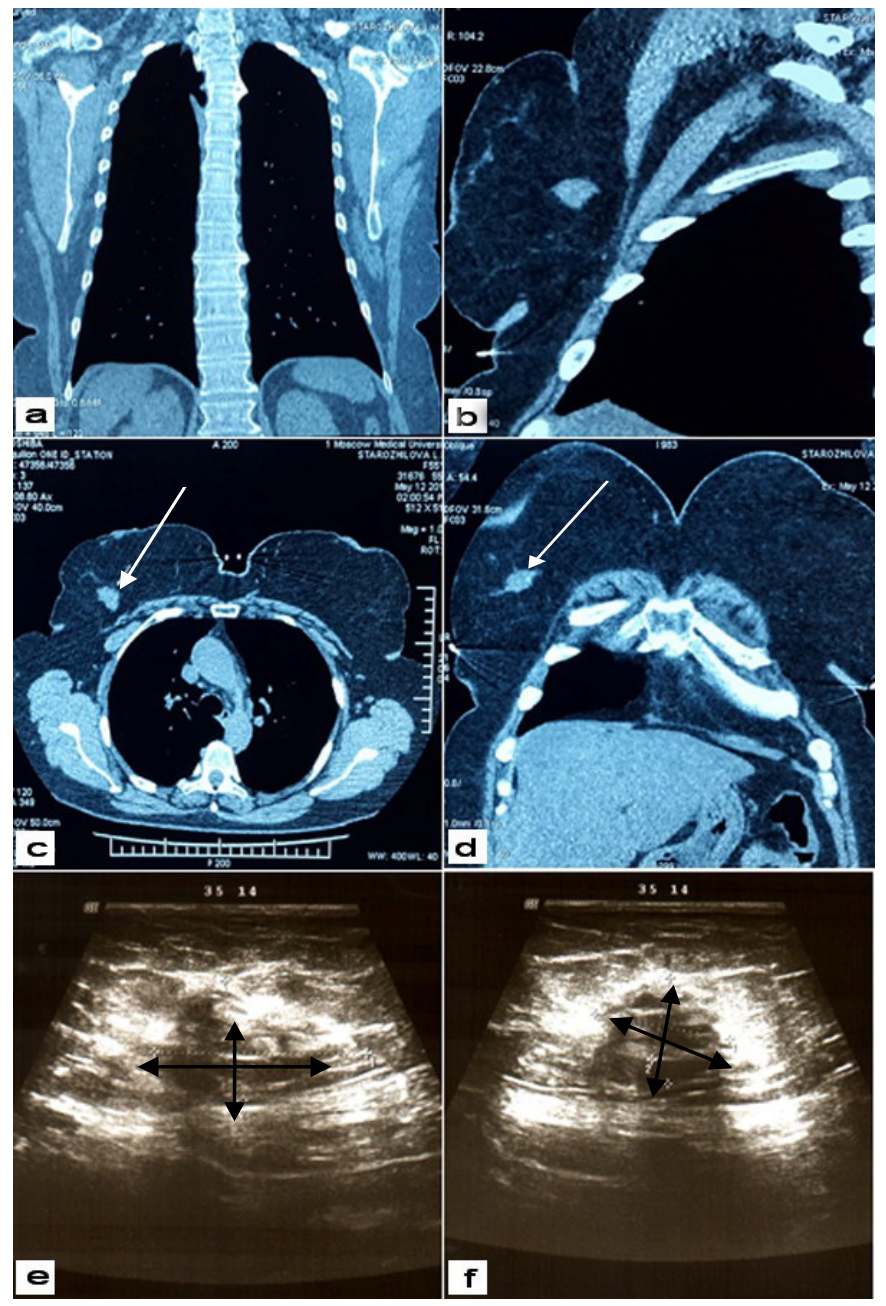

Fig. 1.

The data of CT scan (a-d) and ultrasonography of the right breast (e-f). Note a large, solid mass (black arrow).

The periphery showed haemosiderin-laden macrophages and moderate chronic inflammatory infiltrate (Fig. 3a-e). Malignant tumor growth was absent in resected material. The final histological diagnosis: duct adenoma of right breast with duct ectasia and cholesterol granulomas.

\section{Discussion}

Cholesterol granuloma of the breast is composed of fibrous granulation tissue containing a large number of cholesterol crystals surrounded by foreign-body giant cells. This pathological condition is more common in the middle ear and mastoid process, frequent in parotid gland, lymph nodes, liver, and spleen. However, peritoneum and mammary glands are rarely affected. ${ }^{(1)}$

The pathogenesis of breast cholesterol granuloma is still unclear, but two theories have been reported. According to the first one, mammary duct ectasia is a disease affecting middle-aged women. ${ }^{(8)}$ The primary disorder is dilatation and stasis of the large duct, with accumulation of fatty detritus in the lumen. If the luminal material escapes from the duct, a florid inflammatory reaction rich in macrophages and plasma cells may ensue. Calcification (often as microcalcification) is common, producing tubular, annular, and linear shadows on a mammogram..$^{(1)}$ The second theory is based on periductal inflammation as a primary lesion, with duct dilatation resulting from damage to the elastic lamina of the duct wall. ${ }^{(8)}$

On one hand, cholesterol granuloma could be caused by periductal inflammation, and cholesterol crystals probably result from lipid-rich material normally found in ectasic ducts escaping from the duct. On the other hand, it can also be the consequence of a rupture of ectasic ducts into the periductal parenchyma due to previous trauma or biopsy (our patient had no history of mammary trauma or biopsy). ${ }^{(5,9,10)}$

The case presented here is the first one where the mammary duct adenoma represents a background process for cholesterol granuloma. Our finding is in accord with both theories of pathogenesis, due to the presence of ductal ectasia with epithelial hyperplasia and periductal inflammation.

The most important aspect is the process of differentiating between cholesterol granuloma and breast carcinoma, due to the lack of clinical and mammographic features of cholesterol granuloma. ${ }^{(4,5,10-12)}$ For instance, clinical examination of cholesterol granuloma, as well as ductal carcinoma, revealed a firm, mobile mass not associated with skin findings, no abnormal nipple discharge, and an absence of axillary or supraclavicular lymphadenopathy. Mammography revealed a high-density shadow with a partly ill-defined margin and calcifications. Although fine needle aspiration biopsy and smears are less expansive and well tolerated by the patient, these methods do not suffice to render a diagnosis because of the scarcity of the material, or they contain scanty inflammatory cells mixed with very few epithelial cells, as in cholesterol granuloma. Therefore, a definite diagnosis cannot be made. It might be more useful to perform a core needle biopsy that provides a tissue core for histologic diagnosis. Moreover, the cytological examination is not sufficient to exclude a carcinomatous lesion.

Macroscopically, the cut surface of granuloma revealed a nodule that was cystic and firm, with yellow concentric ring-fingers, whereas a ductal carcinoma of the breast shows irregular, stellate or nodular configuration. However, some authors have reported cholesterol granulomas more than $2 \mathrm{~cm}$ in diameter with papillary structures. ${ }^{(6)}$ 

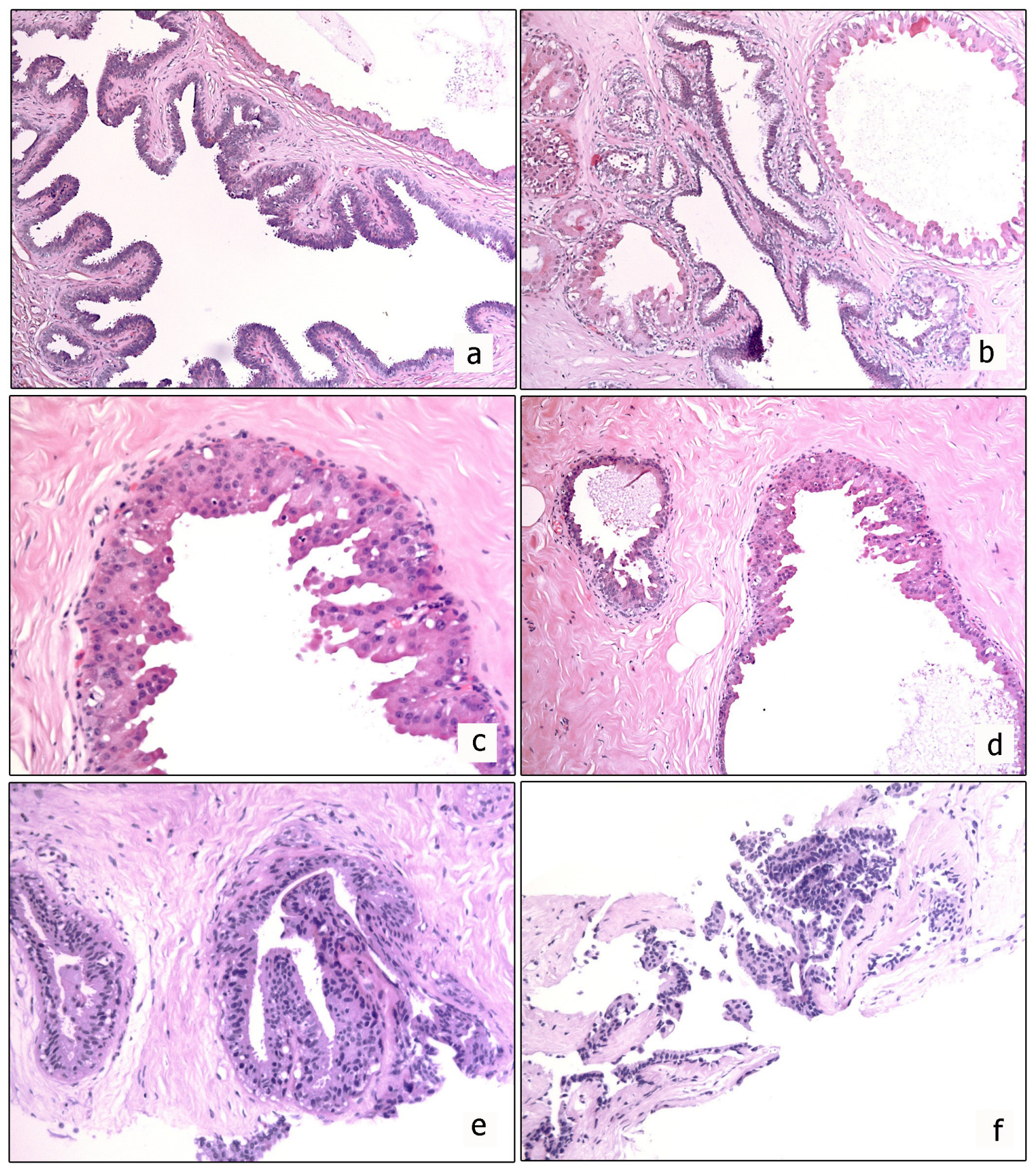

Fig. 2. Ductal adenoma of the breast.

a-d - surgical material; e-f - biopsy. a - duct with papillary configuration (H\&E; magnification: $\times 10) ; b-$ ducts with epithelial proliferation and apocrine change (H\&E; magnification: $\times 10)$; $c$-apocrine changes of the ductal epithelium (H\&E, magnification: $\times 20)$; $d$ - the elaboration of the apocrine metaplasia (H\&E; magnification: $\times 10) ; e-$ gland with epithelial proliferation and cribriform structure; nucleus are in the different levels (H\&E, magnification: $\times 20) ; f$ - noncohesive cells of similar type, the structure of the gland is not defined, perhaps the focus of infiltrative growth (H\&E; magnification: $\times 20)$. 

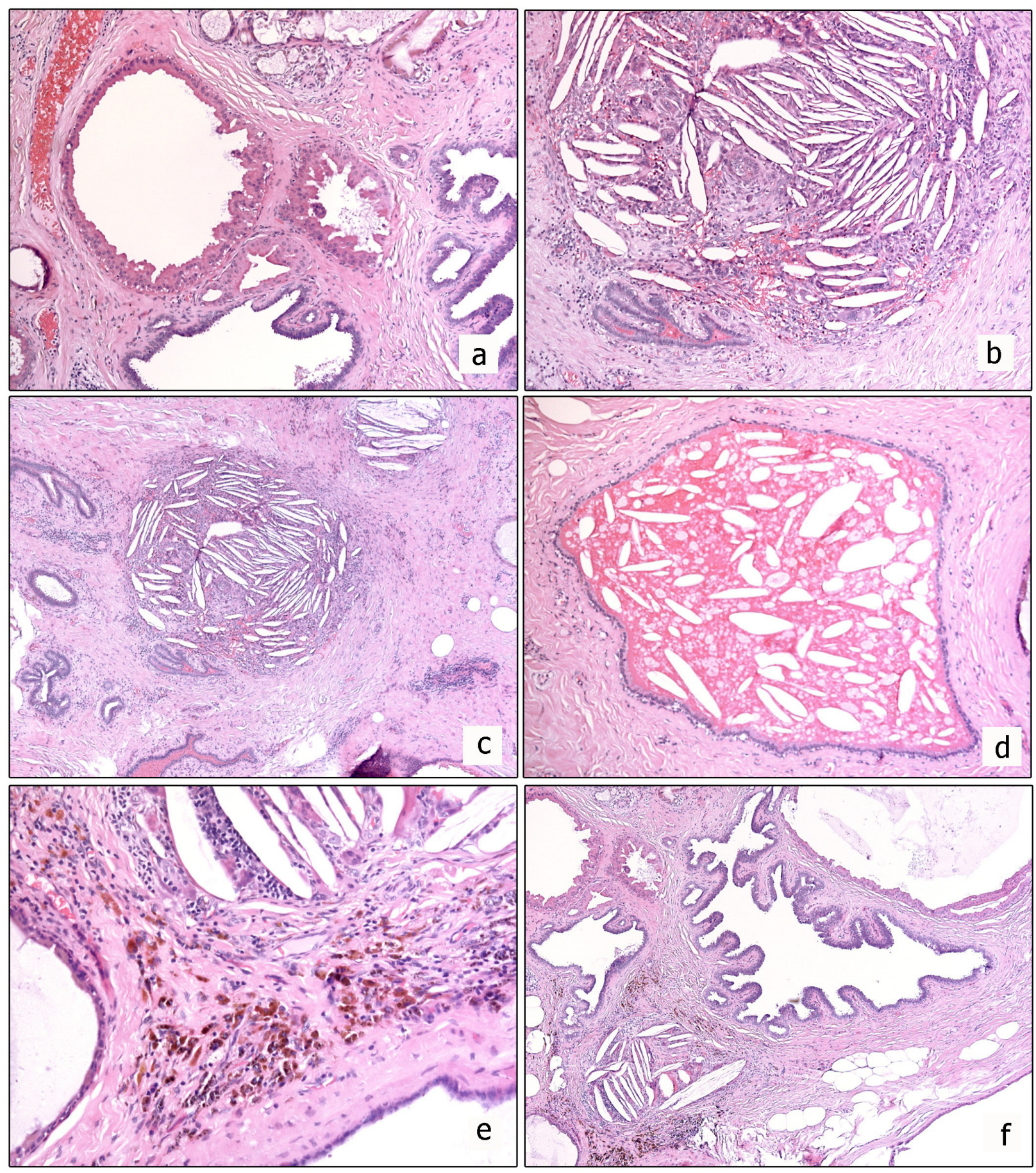

Fig. 3. The breast cholesterol granuloma.

$a$ - the cholesterol granuloma between the breast ducts (H\&E; magnification: $\times 5)$; $b$ cholesterol clefts in fibrotic interstitium with surrounding chronic inflammatory infiltrate and foreign body type multinucleated giant cells (H\&E; magnification: $\times 5)$; c - cholesterol clefts with surrounding chronic inflammatory infiltrate and foreign body type multinucleated giant cells (H\&E; magnification: $\times 10)$; $d$-dilated duct lumen contains many needlelike cholesterol crystals (H\&E; magnification: $\times 10$ ); $e$-in the periphery of granuloma-deposites of hemosiderine (H\&E; magnification: $\times 20) ; f$-duct ectasia with apocrine metaplasia, papillary structure and periductal inflammation (H\&E; magnification: $\times 10)$. 
Differential diagnosis from papillary neoplasms is based on ultrasonographic features, such as the shape and vascularity of the intraductal lesion, and associated findings such as ductal dilatation. The latter appears as an intracystic solid lesion with peripheral fronting in the dilated duct, with secondary ductal dilatation proximal to the mass. Moreover, the intracystic solid lesion showed increased vascularity. ${ }^{(13)}$ Blood chemistry reveals high cholesterol and triglyceride levels. Although this sign is not specific, especially in patients with hypercholesterolemia, atherosclerosis or other disorders of lipid metabolism.

Histologically, it is not difficult to distinguish between cholesterol granuloma and breast carcinoma. Granuloma of the breast has a typical aspect of needle-like crystals arranged in parallel or radial arrays, sometimes with microcalcifications and metaplastic unusual bone surrounded by histiocytes and giant cells. However, the background process for cholesterol granuloma, such as epithelial hyperplasia and apocrine metaplasia with intraductal papillary structures, could be mistaken for a malignant process. ${ }^{(1,14)}$ Although cholesterol granuloma is not claimed as a risk factor associated with cancer, these conditions progress parallel to each other. Therefore, good histological analysis is required because cancer can be associated with cholesterol granuloma of the breast.

We conclude that breast cholesterol granuloma is a rare lesion that might be accompanied by osseous metaplasia. It must be suspected in woman that carry no risk factors for breast cancer, and an accurate, preoperative study must also be conducted using core needle biopsy, which is more sensitive than fine needle biopsy. Even if surgery is necessary, it must be conservative, and the pathologist must perform a good macroscopic and histological analysis to recognize, eventually, an unknown associated cancer.

There is a tendency to believe that cholesterol granulomas in the lungs have mutual origins with cholesterol granulomas in the breast. These are extremely rare conditions unrelated to endogenous lipoid pneumonia, pulmonary alveolar proteinosis, or cholesterol pneumonia, which could be found during pneumectomy or autopsy. On the one hand, pulmonary parenchymal cholesterol granulomas have been reported in patients with known pulmonary hypertension with the conclusion that a severe increase in pulmonary blood pressure was most important in the pathogenesis. ${ }^{(15,16)}$ On the other hand, cholesterol granulomas could be secondary to or independent of pulmonary hypertension. ${ }^{(17)}$

Histopathologic examination of 36 patients with plexogenic pulmonary arteriopathy revealed cholesterol granulomas in 9 cases. ${ }^{(18)}$ In another series of radiographic and histopathologic examinations of 20 patients with pulmonary hypertension, cholesterol granulomas were found in 5 cases, but no association with plexiform lesions was suggested. ${ }^{(19)}$

Another patient who developed cholesterol granulomas after busulfan treatment for chronic myeloid leukemia, was postulated to be secondary to pneumocyte desquamation and independent of pulmonary hypertension. ${ }^{(20)}$ Although microangiopathic hemolytic anemia and thrombocytopenia have been associated with primary pulmonary hypertension, they have not been linked to the development of granulomatous lesions with cleft-like cholesterol deposits.

One of the possible pathogenetic factors is the lysis of red blood cells with release of their membrane lipids. ${ }^{(16)}$ Microangiopathic hemolytic anemia and thrombocytopenia are in association with primary pulmonary hypertension, and the consumption of erythrocytes and platelets was attributed to their destruction in plexiform lesions. ${ }^{(21)}$ In this case, plexiform lesions most likely developed secondary to longstanding pulmonary hypertension, causing hemolysis and thrombocytopenia. Destruction of red blood cells and platelets in plexiform lesions could release membrane lipids and provide them for uptake by multinucleated phagocytes, which could later form granulomas. The presence of hemosiderin granules within those cells supports this hypothesis. Therefore, chronic hemolytic anemia and thrombocytopenia might be contributing factors to the development of cholesterol granuloma. There are reliable data about increased (18)F-fluorodeoxyglucose uptake with cholesterol granuloma during PET-CT examination. ${ }^{(22)}$ Therefore, our patient's reported history of thrombocytopenia, arterial hypertension and severe hypercholesterolemia, could increase the possibility of revealing cholesterol granulomas in the lungs.

Mammary cholesterol granuloma is an extremely rare benign condition affecting middle-aged women with duct ectasia as a background process. There are no data about duct adenoma of the breast as a background process for cholesterol granuloma. This condition is not claimed as a risk factor associated with cancer, although on clinical examination these diseases are difficult to differentiate. There are several cases with cholesterol granuloma and carcinoma that progressed parallel to each other. The described case is noticeable by the combination of multiple cholesterol granulomas in the lungs (with arterial hypertension and thrombocytopenia as a background) with breast presentation, which was associated ductal adenoma.

\section{Competing interests} interests.

The authors declare that they have no competing

\section{References}

1. Garofalo S, Casolino C, Accurso A, Falleti J. Cholesterol granuloma of the breast with unusual ossification features (osseous metaplasia). Pathol Res Pract. 2008;204(5):353-6. doi:10.1016/j.prp.2007.12.004.

2. Bezic J, Piljic-Burazer M. Breast cholesterol granuloma: a report of two cases with discussion on potential pathogenesis. Pathologica. 2013;105(6):349-52.

3. Ishizaki M, Ohsumi S, Takashima S, Mandai K. Two cases of cholesterol granuloma of the breast. Breast Cancer. 2001;8(2):158-61.

4. Khan R, Narula V, Jain A, Maheshwari V. Cholesterol granuloma of the breast mimicking malignancy. BMJ Case Rep. 2013;2013. pii: bcr2013200108. doi:10.1136/bcr-2013200108.

5. Reynolds HE, Cramer HM. Cholesterol granuloma of the breast: a mimic of carcinoma. Radiology. 1994;191(1):249-50. 
6. Smith GL, Hicks P, Wijesinghe DP, Holme TC. Cholesterol granuloma of the breast presenting as an intracystic papilloma. Br J Radiol. 1997;70(839):1178-9.

7. Furuhira C1, Ohshima A, Shimada K, Kuroki S, Nakano $\mathrm{K}$, Ishikawa $\mathrm{M}$, et al. A case of breast cholesterol granuloma accompanied by cancer. Breast Cancer. 2004;11(2):210-3.

8. Dixon JM, Anderson TJ, Lumsden AB, Elton RA, Roberts MM, Forrest AP. Mammary duct ectasia. Br J Surg. 1983;70(10):601-3.

9. Ahn HS, Kim SM, Yun BL, Kim MS, Jang M, Park SY, et al. The unusual ultrasound features of a breast cholesterol granuloma manifesting as an intracystic mass: case report and literature review. Korean J Radiol. 2013;14(2):179-82. doi:10.3348/kjr.2013.14.2.179.

10. Osada T, Kitayama J, Nagawa H. Cholesterol granuloma of the breast mimicking carcinoma: report of a case. Surg Today. 2002;32(11):981-4.

11. Eichner H, Heymer B. [Cholesterol granuloma (so-called cholesteatoma) of the breast. The differential diagnosis of a slowly growing cancer]. Rofo. 1987;146(4):464-6. [Article in German].

12. Wilhelmus JL, Schrodt GR, Mahaffey LM. Cholesterol granulomas of the breast. A lesion which clinically mimics carcinoma. Am J Clin Pathol. 1982;77(5):592-7.

13. Ganesan S, Karthik G, Joshi M, Damodaran V. Ultrasound spectrum in intraductal papillary neoplasms of breast. $\mathrm{Br} \mathrm{J}$ Radiol. 2006;79(946):843-9.

14. Perino A, Petronio M. [Granulomatous cholesterol salpingitis with ceroid accumulation and osseous metaplasia].
Acta Eur Fertil. 1974;5(3):259-74. [Article in English, Italian]. 15. Cholesterol granuloma of the lung. $\mathrm{Br}$ Med $\mathrm{J}$. 1969;1(5641):396.

16. Glancy DL, Frazier PD, Roberts WC. Pulmonary parenchymal cholesterol-ester granulomas in patients with pulmonary hypertension. Am J Med. 1968;45(2):198-210.

17. Fischer EG, Marek JM, Morris A, Nashelsky MB. Cholesterol granulomas of the lungs associated with microangiopathic hemolytic anemia and thrombocytopenia in pulmonary hypertension. Arch Pathol Lab Med. 2000;124(12):1813-5.

18. Caslin AW, Heath D, Madden B, Yacoub M, Gosney JR, Smith P. The histopathology of 36 cases of plexogenic pulmonary arteriopathy. Histopathology. 1990;16(1):9-19.

19. Nolan RL, McAdams HP, Sporn TA, Roggli VL, Tapson VF, Goodman PC. Pulmonary cholesterol granulomas in patients with pulmonary artery hypertension: chest radiographic and CT findings. AJR. Am J Roentgenol. 1999;172(5):1317-8.

20. Kay JM, Heath D, Hasleton PS, Littler WA. Aetiology of pulmonary cholesterol-ester granulomas. $\mathrm{Br} \mathrm{J}$ Dis Chest. 1970;64(1):55-7.

21. Jubelirer SJ. Primary pulmonary hypertension. Its association with microangiopathic hemolytic anemia and thrombocytopenia. Arch Intern Med. 1991;151(6):1221-3.

22. Wada H, Sakashita T, Shigemori W, Nagao T, Nakano Y. A case of pulmonary cholesterol granuloma: an (18) F-fluorodeoxyglucose positron emission tomography image. Am J Med Sci. 2012;344(5):414-5. doi:10.1097/ MAJ.0b013e3182583196. 